\title{
The Wave Properties of Matter: The Physical Aspect
}

\author{
Liudmila B. Boldyreva* \\ The State University of Management, Moscow, Russia \\ *Corresponding author: boldyrev-m@yandex.ru
}

Received September 20, 2014; Revised September 27, 2014; Accepted October 27, 2014

\begin{abstract}
The aim of the paper is to show that there is a physical process which could underlie the wave properties of matter. A comparison has been drawn between the properties of a pair of electrically unlike virtual particles created by a quantum entity in the physical vacuum and the characteristics of the quantum entity wave function. Analogies were revealed between the spin precession frequency of pair of virtual particles and the wave function frequency, between the size of the electric dipole produced by a pair of virtual particles and the wave function wavelength, and also between the angle of spin precession of pair of virtual particles and the wave function phase. It is shown that quantum correlations of quantum entities may be caused by spin correlations (by spin supercurrents) between virtual particles created by the quantum entities in the physical vacuum. It is shown that the wave properties of a quantum entity are due to precession of spin of pair of virtual particles created by the quantum entity in the physical vacuum.
\end{abstract}

Keywords: wave of matter, quantum electric dipole moment, wave function frequency, wave function wavelength, virtual particles, quantum correlations, spin supercurrent

Cite This Article: Liudmila B. Boldyreva, "The Wave Properties of Matter: The Physical Aspect." International Journal of Physics, vol. 2, no. 6 (2014): 189-196. doi: 10.12691/ijp-2-6-2.

\section{Introduction}

In 1923 de Broglie [1] put forward a hypothesis that with every particle of nonzero rest mass a wave is associated. Over the next several decades efforts were focused on searching of a "physical" wave that could accompany the particle: the so-called pilot wave. $[2,3]$ The only quantum entity whose wave function characteristics have a physical meaning is the photon. For the photon the wave function frequency and wavelength are the photon's frequency and wavelength respectively. As concerns quantum entities with nonzero rest mass, there is something like a physical meaning only for the wavelength: the latter determines the size of the quantum entity with due account of its wave properties. There are two wave functions: the Schrödinger wave function $\psi_{S h}(\mathbf{x}, t)$ and the de Broglie wave function $\psi_{B}(\mathbf{x}, t)$, which are used for description of wave properties of quantum entities in the nonrelativistic approximation. For the freely moving (in the absence of external forces) quantum entity possessing momentum $\mathbf{p}$ and mass $m_{0}$ we have [2]:

$$
\begin{gathered}
\psi_{B}(\mathbf{x}, t)=\exp \left(i \mathbf{x k}-i \omega_{B} t\right), \\
\psi_{S h}(\mathbf{x}, t)=\exp \left(i \mathbf{x k}-i \omega_{S h} t\right),
\end{gathered}
$$

where $\mathbf{x}$ and $t$ are the positional coordinate and time respectively; $\mathbf{k}$ is the wave vector, $\omega_{B}$ is the de Broglie wave function frequency, $\omega_{S h}$ is the Schrödinger wave function frequency.

$$
\mathbf{k}=\frac{\mathbf{p}}{\hbar}=\frac{2 \pi \mathbf{p}}{\lambda p}
$$

where $\lambda$ is the wavelength of wave function:

$$
\lambda=2 \pi \hbar / p,
$$

where $\hbar$ is Planck's constant.

$$
\begin{gathered}
\omega_{B}=\frac{p^{2}}{2 m_{0} \hbar}+\frac{m_{0} c^{2}}{\hbar}, \\
\omega_{S h}=\frac{p^{2}}{2 m_{0} \hbar} .
\end{gathered}
$$

The phase $\varphi_{B}(\mathbf{x}, t)$ associated with the wave function $\psi_{B}(\mathbf{x}, t)$, and phase $\varphi_{S h}(\mathbf{x}, t)$ associated with the wave function $\psi_{S}(\mathbf{x}, t)$ are determined respectively, according to Eqs. (1), (2) and (3), as

$$
\begin{gathered}
\varphi_{B}(x, t)=\mathbf{x k}-\omega_{B} t, \\
\varphi_{S h}(x, t)=\mathbf{x k}-\omega_{S h} t .
\end{gathered}
$$

The phase speed $\left(\boldsymbol{v}_{f}\right)_{B}$ associated with the wave function $\psi_{B}(\mathbf{x}, t)$ is determined, according to Eqs. (3), (5) and (7), as

$$
\left(\mathbf{v}_{f}\right)_{B}=\frac{\omega_{B} \mathbf{k}}{k^{2}}=\left(\frac{p}{2 m_{0}}+\frac{m_{o} c^{2}}{p}\right) \frac{\mathbf{p}}{p},
$$


where $c$ is the speed of light. The phase speed $\left(\boldsymbol{v}_{f}\right)_{S h}$ associated with the wave function $\boldsymbol{\psi}_{S h}(\mathbf{x}, t)$ is determined, according to Eqs. (3), (6) and (8), as

$$
\left(\mathbf{v}_{f}\right)_{S h}=\mathbf{p} /\left(2 m_{0}\right) \text {. }
$$

It is seen from Eqs. (3)-(10) that only the wavelength $\lambda$ of quantum entity is determined in the same way both for the de Broglie wave function $\psi_{B}(\mathbf{x}, t)$ and for the Schrödinger wave function $\boldsymbol{\psi}_{S h}(\mathbf{x}, t)$. Other wave function characteristics: the frequency, phase and phase speed are determined differently for $\boldsymbol{\psi}_{S h}(\mathbf{x}, t)$ and $\boldsymbol{\psi}_{B}(\mathbf{x}, t)$. Since both wave functions describe the same quantum entity, it was accepted in quantum theory that the frequency, phase and phase speed of the wave function describing quantum entities with nonzero rest mass have no physical meaning, that is, they are not characteristics of oscillations of any physical process.

With respect to the wave function, the Born proposal has been accepted, whose main idea consists in that only the square of the absolute value of the wave function has the physical meaning: It determines the probability density of finding the particle at a point of space. From this point of view, there is no contradiction between the Schrödinger and de Broglie wave functions: they describe the same probability density of finding a particle at a point of space. That is, according to Eqs. (1) and (2): $\left|\psi_{S}(\mathbf{x}, t)\right|^{2}=\left|\psi_{B}(\mathbf{x}, t)\right|^{2}$.

In addition to the wave properties, quantum entities are able to produce virtual particles in the physical vacuum. [4] The virtual particles account for the following effects: the spontaneous emission of photon, Casimir effect, quantum fluctuations in a vacuum, vacuum polarization, production of electron-positron pairs, Lamb shift and others. The virtual particles have the following features:

1. A pair of virtual particles is created by a quantum entity in the region, whose size is of the order of magnitude of the wavelength of the quantum entity.

2. A pair of virtual particles is characterized by the energy, $U_{v}$. The energy conservation law holds with the uncertainty $\Delta U_{v}$ associated with the uncertainty of lifetime of virtual particles $\Delta t$ by relation $\Delta U_{v} \sim \hbar / \Delta t$; at $\Delta t \rightarrow \infty$ it follows that $\Delta U_{v} \rightarrow 0$.

3. A pair of virtual particles may convert into a pair of real particles if the energy of virtual pair becomes equal to or exceeds energy $2 m_{0} c^{2}$, where $m_{0}$ is the mass of the real particle produced. For example, if the energy of the pair of virtual particles created by a photon becomes equal to the total energy of some real particle-antiparticle pair (for example, electron-positron pair), then the pair of virtual particles is converted into the real particleuntiparticle pair.

4. A pair of virtual particles has a mass $m_{v}$ related to the energy of the pair, $U_{v}$, by the classical equation

$$
m_{v}=U_{v} / c^{2} .
$$

5. The electric properties of virtual particles are the same as those of real particles. Consequently, a pair of electrically unlike virtual particles is an electric dipole whose electric properties are the same as those of the electric dipole produced by electrically unlike real particles.

6. The angular momentum conservation law holds, and the virtual particle spin has the same properties as the real particle spin. Hence it follows that:

a) the spin of pair of virtual particles has no definite direction, and by the magnitude of spin the magnitude of its projection onto a preferential direction is meant; this can be interpreted as a precession of the spin about the preferential direction. The precession is characterized by precession and nutation angles, and by precession frequency $\omega_{v}$ which is defined at small $\beta$ [5] as:

$$
U_{v}=S_{v} \omega_{v}
$$

b) spin correlations may take place between the spins of pairs of virtual particles.

It is shown in this paper that the wave properties of a quantum entity are due to creation of pairs of virtual particles by quantum entities in the physical vacuum. The frequency of Schrödinger's wave function is the frequency of precession of spin of the pair of virtual particles created by the quantum entity. The wave function wavelength is equal to $2 \pi$-fold size of electric dipole produced by a pair of virtual particles created by the quantum entity. The phase of wave function is associated with the precession angle of spin of the pair of virtual particles created by the quantum entity in the physical vacuum. The comparison of the properties of wave function and those of pairs of virtual particles enables one to provide a physical interpretation for such a phenomenon as quantum correlation of quantum entities described by the same wave function $[6,7]$.

\section{The Frequency of Wave Function of Quantum Entities}

The pairs of virtual particles produced by photons in the physical vacuum are converted into real particles (electron-positron pairs, proton-antiproton pairs), if the energy of the photon equals the total energy of the pair of real particles produced. This means that the energy of a pair of virtual particles produced by the photon in the physical vacuum $\left(U_{v}\right)_{p h}$ is equal to the energy of photon, $U_{p h}:$

$$
\left(U_{v}\right)_{p h}=U_{p h}
$$

and the total spin of the pair of virtual particles produced by a photon, $\left(S_{v}\right)_{p h}$, equals:

$$
\left(S_{v}\right)_{p h}=\hbar \text {. }
$$

Comparing Eqs. (12)-(14) with the well-known relation between energy $U_{p h}$ and photon frequency $\omega_{p h}$ : $U_{p h}=\hbar \omega_{p h}$, we obtain:

$$
\left(\omega_{v}\right)_{p h}=\omega_{p h},
$$


where $\left(\omega_{v}\right)_{p h}$ is the precession frequency of the spin of pair of virtual particles produced by the photon in the physical vacuum.

Assuming that the equation similar to Eq. (13) is valid for any quantum entity, we have:

$$
\left(U_{v}\right)_{q}=U_{q},
$$

where $U_{q}$ is the energy of quantum entity, $\left(U_{v}\right)_{q}$ is the energy of the pair of virtual particles produced by the quantum entity in the physical vacuum. According to quantum theory, electric interactions of electrically charged real particles are effected through virtual photons, which are essentially pairs of electrically unlike virtual particles. This means that at least for spin $\left(S_{v}\right)_{q}$ of the pair of electrically unlike virtual particles produced by an electrically charged particle in the physical vacuum the following holds:

$$
\left(S_{v}\right)_{q}=\hbar
$$

Comparing Eqs. (12), (16) and (17) with the wellknown relation between energy of quantum entity $U_{q}$ and frequency of its Schrödinger wave function, $\omega_{S h}$, i.e. [2]:

$$
U_{q}=\hbar \omega_{S h},
$$

we obtain:

$$
\left(\omega_{v}\right)_{q}=\omega_{S h}
$$

where $\left(\omega_{v}\right)_{q}$ is the precession frequency of the spin of pair of virtual particles produced by the quantum entity in the physical vacuum.

Therefore, according to Eqs. (15) and (19), the frequency of the wave function for a quantum entity (for the entity with non-zero rest mass this is the Schrödinger wave function) equals the precession frequency of spin of the pair of virtual particles produced by the quantum entity in the physical vacuum.

\subsection{The Mass Determined by the Wave Function Frequency}

The so-called kinetic mass, $m_{p h}$, is associated with the photon, the mass being determined by the photon frequency: $m_{p h}=\hbar \omega_{p h} / c^{2}$, or taking into account Eq. (15):

$$
m_{p h}=\hbar\left(\omega_{v}\right)_{p h} / c^{2}
$$

The so-called relativistic increase of mass, $\Delta m$, is associated with moving particles, the increase being derived in special relativity [8]: $\Delta m=m_{0}\left|(v / c)^{2} / 2+o\left((v / c)^{2}\right)\right|$, where $o\left((v / c)^{2}\right)$ is an addend of less order of magnitude than $(v / c)^{2}, v$ and $m_{0}$ are the speed and rest mass of the quantum entity respectively. Correct to the second order of $\beta=v / c$ the following holds:

$$
\Delta m=m_{0} v^{2} /\left(2 c^{2}\right) .
$$

In the case where the energy of a quantum entity, $U_{q}$, is equal to its kinetic energy

$$
U_{q}=m_{0} v^{2} / 2
$$

and using Eqs. (18), (19) and (22) in (21), we obtain:

$$
\Delta m=\hbar\left(\omega_{v}\right)_{q} / c^{2} .
$$

From the comparison of Eqs. (14), (17), (20) and (23) with Eqs. (11) and (12) it follows that the kinetic mass of photon and the relativistic increase of mass of quantum entity with nonzero rest mass are the mass of the pair of virtual particles created by the quantum entity in the physical vacuum.

Note. It follows from Eqs. (18) and (22) that $v=\sqrt{2 \hbar \omega_{S h} / m_{0}}$. It is noteworthy that both velocity and frequency are vectors. Thus a change in the magnitude and/or direction of the velocity of a quantum entity results in a change in the magnitude and/or direction of the precession frequency of spin of the pair of virtual particles produced by the quantum entity in the physical vacuum. Note that any quantum entity with mass $m_{0}$ has been once generated from a virtual particle when the energy of the latter became equal to $m_{o} c^{2}$. Thus inertial properties of any quantum entity may be associated with gyroscopic properties of the virtual particle that generated the entity and with gyroscopic properties of the pair of virtual particles being created by the entity in the physical vacuum.

\section{The Wavelength of Wave Function}

Below we shall specificate the notion of the wavelength of wave function. To this end we shall specify the electric dipole moment of a pair of electrically unlike virtual particles created by a quantum entity as a function of characteristics of the wave function of the entity. Hereafter we shall call this moment "the quantum electric dipole moment - QEDM”.

\subsection{Quantum Electric Dipole Moment}

To determine QEDM, $d_{v}$, of a quantum entity let us introduce the following designations: $q_{v}$ is the charge of a virtual particle, $L$ is the distance between virtual particles in the pair, $U_{v}$ is the energy of a virtual particle pair. Under the definition of QEDM [9]:

$$
d_{v}=q_{v} L
$$

Let us determine the QEDM for the electron, $\left(d_{v}\right)_{e}$. We assume that the specific charge of the virtual particle produced by an electron is equal to the specific electron charge:

$$
e / m_{e}=2 q_{v} / m_{v}
$$

where $e$ and $m_{e}$ are the electric charge and mass of electron, respectively; factor 2 in the right-hand side of the 
equation is due to the fact that the mass of only one virtual particle of the pair must be used in the equation. Since, according to property 1 from Section 1 , a quantum entity creates a pair of virtual particles in the region whose size is of the same order of magnitude as the wavelength of the quantum entity, we may assume that

$$
L=\gamma \cdot \lambda,
$$

where $\gamma$ is a proportionality coefficient; $\lambda=\lambda /(2 \pi)$ is the wavelength of wave function. Using equations (11), (12), (17), (24)-(26) and the expression for Bohr's magneton $\mu_{B}=e \hbar /\left(2 m_{e} c\right)$, QEDM can be expressed in terms of the wavelength and frequency of the wave function of the quantum entity:

$$
\left(d_{v}\right)_{e}=\gamma \mu_{B} \lambda \omega_{S h} / c .
$$

Using the relation between $\omega_{S h}$ and energy of electron, $U_{e}, U_{e}=\hbar \omega_{S h}$, and also the relation between $\lambda$ and the electron momentum $p_{e}$ :

$$
\bar{\lambda}=\hbar / p_{e}
$$

expression (27) for $\left(d_{v}\right)_{e}$ can be written as:

$$
\left(d_{v}\right)_{e}=\frac{\gamma \mu_{B} U_{e}}{c p_{e}} .
$$

At $U_{e}=m_{e} v^{2} / 2$ ( $v$ is the speed of electron), equation (29) takes the form:

$$
\left(d_{v}\right)_{e}=\frac{\gamma \mu_{B} v}{2 c}
$$

It is obvious that Eqs. (29) and (30) are valid as well for the positron's QEDM, $\left(d_{v}\right)_{p}$.

Let us consider the mutual orientation of velocity $\mathbf{v}$ and dipole moment $\mathbf{d}_{v}$. According to Eq. (26), the size of the dipole produced in the physical vacuum by a moving quantum entity is determined by the wavelength of its wave function. Taking into account Eq. (3) we can assume that $\mathbf{d}_{v} \uparrow \uparrow \mathbf{k}$ or $\mathbf{d}_{v} \uparrow \downarrow \mathbf{k}$. If the quantum entity has an electric charge, its electric field acts as a moment on the electric dipole created by the quantum entity. Therefore, if $\mathbf{p} \uparrow \uparrow \boldsymbol{v}$, then depending on the sign of the quantum entity charge the QEDM created by the entity is aligned with the quantum entity velocity or directed oppositely. Thus, for example, for the electron $\left(\mathbf{d}_{v}\right)_{e} \uparrow \uparrow \boldsymbol{v}$, for the positron $\left(\mathbf{d}_{v}\right)_{p} \uparrow \downarrow v$. In the general case:

$$
\mathbf{d}_{v} \uparrow \downarrow \mathbf{I},
$$

where I is the electric current created by the moving charged quantum entity. Mutual orientation of velocity $\boldsymbol{v}_{e}$ and QEDM $\left(\mathbf{d}_{v}\right)_{e}$ for electron (e), and the same for velocity $\boldsymbol{v}_{p}$ and QEDM $\left(\mathbf{d}_{v}\right)_{p}$ for positron $(p)$ are shown on Figure 1.

To determine coefficient $\gamma$ let us consider the electron in a hydrogen atom. In the electric field $\mathbf{E}_{n}$ of the nucleus a moment $\mathbf{M}$ will act on the electron caused by QEDM of the electron [9]: $\mathbf{M}=\left(\mathbf{d}_{v}\right)_{e} \times \mathbf{E}_{n}$.
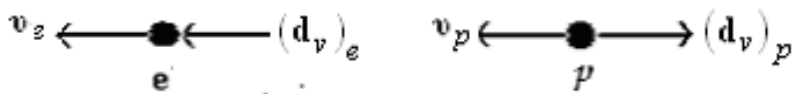

Figure 1. Mutual orientation of velocity $\boldsymbol{v}_{e}$ and QEDM $\left(\mathbf{d}_{v}\right)_{e}$ for electron (e); the same for velocity $\mathbf{v}_{p}$ and QEDM $\left(\mathbf{d}_{v}\right)_{p}$ for positron (p)

If the atom is in the ground state, the energy of the electron will be equal to its kinetic energy. In this case Eq. (30) is valid, and the expression for $M$ will take the form: $\mathbf{M}=\frac{\gamma \mu_{B}}{2 c}\left(\mathbf{v} \times \mathbf{E}_{n}\right)$. The right side of the expression for $\mathbf{M}$ coincides with the expression for the spin-orbit interaction energy for an electron in an atom [10]: $U_{s-o}=-\frac{\boldsymbol{\mu}_{\mathbf{B}}}{2 c}\left(\mathbf{E}_{n} \times \boldsymbol{v}\right)$, under $\boldsymbol{\mu}_{B} \uparrow \uparrow\left(\mathbf{E}_{n} \times \boldsymbol{v}\right)$ and

$$
\gamma=1
$$

Then expression (29) for $\left(d_{v}\right)_{e}$ takes the form:

$$
\left(d_{v}\right)_{e}=\frac{\mu_{B} U_{e}}{c p_{e}} .
$$

Thus, if the spin-orbit interaction of an atomic electron and the nucleus is determined by the moment acting in the nucleus electric field on the dipole produced by the electron, then, according to Eqs. (26) and (32), the wavelength divided by $2 \pi$ (e.g. $\lambda$ ) of quantum entity wave function equals the size of electric dipole formed by the pair of virtual particles created by the quantum entity in the physical vacuum.

Note. It is of interest that application of Eq. (33) to the QEDM of photon, $d_{p h}$, results in $d_{p h}=\mu_{B}$.

To verify expression (33) for $\left(d_{v}\right)_{e}$ let us consider examples of using the equation for deriving experimentally proven relationships describing the following phenomena:

- the interaction between two parallel uncharged conducting plates (the Casimir effect);

- the change of the size of a system of electric charges set in motion, the system being in equilibrium under the action of electrostatic forces only.

\subsection{The Interaction between Two Parallel Uncharged Conducting Plates (the Casimir effect)}

There may exist an electric force between two parallel uncharged metal plates owing to QEDM of conduction electrons. If $\mathbf{d}_{1} \uparrow \downarrow \mathbf{d}_{2}$ or $\mathbf{d}_{1} \uparrow \uparrow \mathbf{d}_{2}$, the force $F_{d}$ between the plates is determined [9] as:

$$
\left|F_{d}\right|=\frac{3 d_{1} d_{2}}{r_{c}^{4}},
$$

where $d_{1}$ and $d_{2}$ are the total QEDM of the first and the second plates respectively, $r_{c}$ is the distance between the 
plates, $\mathbf{r}_{C} \perp \mathbf{d}_{1}\left(\mathbf{d}_{2}\right)$. Note that Eq. (34) applies only if the size of dipoles is much less than the distance between them. Taking into account Eqs. (24), (26), (28) and (32), and also the fact that for free electrons in metals $p_{e} \approx 10^{-25} \mathrm{~kg} \mathrm{~m} / \mathrm{s}$ [11], we have: $L \approx 10^{-10} \mathrm{~m}$. In the experiments $r_{c} \approx 10^{-9} \mathrm{~m}$. If the total QEDM of either plate may be determined by mere addition of QEDM of individual electrons and the total size of all dipoles has the same order of magnitude as the size of a single dipole, then the use of Eq. (34) is quite in order.

Let us consider the plates of unit area, taking into account only the electrons present in the $y$ thick layer of the plate surface. If the plates are identical, $d_{1}=d_{2}$. We shall make a rather strong assumption that the QEDM of such electrons in either plate are oriented in the same direction. Then using Eq. (33) in Eq. (34), we have:

$$
F_{d}=\frac{3 \mu_{B}^{2} U_{e}^{2}}{r_{c}^{4} c^{2} p_{e}^{2}} y^{2} N_{0}^{2},
$$

where $N_{0}$ is the conduction electron concentration in metals. The energy of a conduction electron in metals, $U_{e}$, is equal to the Fermi energy $\varepsilon_{F}$; the momentum $p_{e}$ for a conduction electron in metals is determined as $p_{e}=\sqrt{2 m_{e} \varepsilon_{F}}$. Using these relations for Eq. (35), we obtain:

$$
F_{d}=\frac{3 \mu_{B}^{2} \varepsilon_{F}}{r_{C}^{4} c^{2} 2 m_{e}} y^{2} N_{0}^{2} .
$$

The conduction electron concentration in metals, $N_{0}$, is of the order of $10^{22} \mathrm{~cm}^{-3}$, and the Fermi energy is determined by the following equation [11]: $\varepsilon_{F}=\hbar^{2}\left(3 \pi^{2} N_{o}\right)^{2 / 3} /\left(2 m_{e}\right)$. Substituting the right side of the latter equation for $\varepsilon_{F}$ in Eq. (36) as well as substituting the values of all constants and assuming $y$ equal to the atom size of $\approx 10^{-10} \mathrm{~m}$, we obtain: $F_{d}=4 \cdot 10^{-23} / r_{c}^{4}(\mathrm{~N})$. This expression for $F_{d}$ is very close to that determining the Casimir force $F$ acting on a unit area of two parallel electrically neutral metallic plates, the distance between them being not greater than some atomic diameters [12]: $F=\hbar c \pi^{2} /\left(240 r_{c}^{4}\right) \approx 10^{-23} / r_{c}^{4}(\mathrm{~N})$.

The sign of $F_{d}$ depends on the mutual orientation of vectors $\mathbf{d}_{1}$ and $\mathbf{d}_{2}$. At $\mathbf{d}_{1} \uparrow \downarrow \mathbf{d}_{2}, F_{d}$ is an attractive force and at $\mathbf{d}_{1} \uparrow \uparrow \mathbf{d}_{2}$ a repulsive force (the latter case is unstable to disturbances). Thus forces of both attraction and repulsion may exist between two uncharged metallic plates. This is supported by the experimental data, e.g. [12, 13]. The repulsive effect taking place under definite conditions is referred to as the "Casimir-Lifshitz effect".

Note. In [12], an explanation of the Casimir effect was suggested, based on the concept of virtual particles; however, in contrast with the Casimir effect explanation given above, only the energies of virtual particles are taken into account in [12], and no forces between the interacting plates are considered. It is assumed in [12] that the energies of all virtual particles in vacuum, associated with the conducting plates, are added together. This assumption is similar to that used in the above description of Casimir force in this paper: the QEDM of electrons of each plate, which are taken into account in Eq. (35), are directed likewise, i.e. are added together.

\subsection{The Change of the Size of a System of Electric Charges Set in Motion, the System being in Equilibrium under the Action of Electrostatic Forces Only}

It was H. Lorentz who noticed that a system of electric charges, being in equilibrium only under the action of electrostatic forces, when set in motion contracts in the direction of the motion [8]. According to the Lorentz mathematical model, the system size in the direction of motion decreases by factor of $\sqrt{1-v^{2} / c^{2}}$, where $v$ is the speed of the system of charges. (Note that the hypothesis of length contraction in the direction of motion was first advanced by Fitzgerald in 1832 [8]). Let us discuss this phenomenon taking into account the QEDM of a moving charge. For simplicity sake we shall consider a system of two electrically charged quantum entities, the system moving at speed $v$. Let us denote by $q_{1}$ and $d_{1}$ the magnitudes of the charge and QEDM respectively of the first entity and by $q_{2}$ and $d_{2}$ the respective characteristics of the second entity. The force of interaction between the two entities has two components: the force due to interaction between them as electric dipoles, $F_{d}$, and the force due to interaction between them as electric charges, $F_{q}$.

$$
F_{q}=\frac{k_{q} q_{1} q_{2}}{r_{q}^{2}},
$$

where $k_{q}$ is the coefficient determined by the system of units used in the calculations, $r_{q}$ is the distance between the charges.

Let us consider two types of mutual direction of velocities $\boldsymbol{v}_{1}$ and $\boldsymbol{v}_{2} \quad\left(v_{1}=v_{2}=v\right)$ of the quantum entities: along the same straight line (Figure2a), and parallel to each other (Figure $2 b$ ).

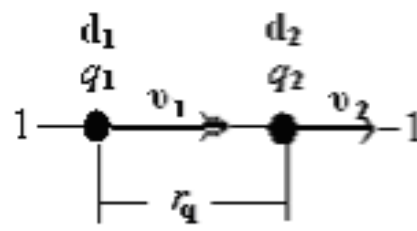

(a)

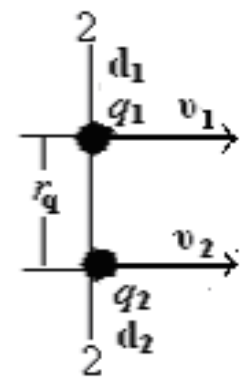

(b)
Figure 2. Variants (a) and (b) for mutual direction of velocities $\boldsymbol{v}_{1}$ and $\boldsymbol{v}_{2}$ of two respective charges $q_{1}$ and $q_{2}$ having QEDM $\mathbf{d}_{1}$ and $\mathbf{d}_{2}$ respectively; $r_{q}$ is the distance between the charges 
Let us determine the resultant force between the two charges for the case of Figure $2 a$. In this case the force $F_{d}$ is expressed as [9]:

$$
F_{d}=\frac{6 d_{1} d_{2}}{r_{q}^{4}}
$$

Taking into account Eq. (31) at any combination of signs of $q_{1}$ and $q_{2}$, the forces $F_{q}$ and $F_{d}$ are directed oppositely, being aligned with the same straight line 1-1. Thus the resultant force $F_{11}$ acting on the charges, according to Eqs. (37) and (38), will be:

$$
F_{11}=\left|F_{q}-F_{d}\right|=\frac{k_{q} q_{1} q_{2}}{r_{q}^{2}}\left|1-\frac{6 d_{1} d_{2}}{k_{q} q_{1} q_{2} r_{q}^{2}}\right| .
$$

Let us determine now the resultant force between the two charges for the variant of their mutual position shown in Figure $2 b$. In this case $F_{d}$ is expressed [9] as:

$$
F_{d}=\frac{3 d_{1} d_{2}}{r_{q}^{4}}
$$

Taking into account Eq. (31) at any combination of signs of $q_{1}$ and $q_{2}$, the forces $F_{q}$ and $F_{d}$, the latter being determined by Eq. (40), have the same direction, being aligned with the same straight line 2-2 which is normal to velocity v . According to Eqs. (37) and (40), the resultant force $F_{22}$ acting on the charges normally to velocity $v$ will be:

$$
F_{22}=\left|F_{q}+F_{d}\right|=\frac{k_{q} q_{1} q_{2}}{r_{q}^{2}}\left|1+\frac{3 d_{1} d_{2}}{k_{q} q_{1} q_{2} r_{q}^{2}}\right| .
$$

Thus the motion of electric charges of any sign results in a decrease of the electric force acting between the charges in the direction of motion, with the reduction factor, according to Eq. (39), of $1-\frac{6 d_{1} d_{2}}{k_{q} q_{1} q_{2} r_{q}^{2}}$, and results in an increase of the force acting in the normal direction, with the amplification factor, according to Eq. (41), of $1+\frac{3 d_{1} d_{2}}{k_{q} q_{1} q_{2} r_{q}^{2}}$. If either of the moving charges is an electron or positron and the energies of the moving charges are equal to their kinetic energies, then, according to Eqs. (30), (32), (39) and (41), the reduction factor is $1-\beta^{2} \frac{6 \mu_{B}^{2}}{4 r_{q}^{2} e^{2}}$ and amplification factor is $1+\beta^{2} \frac{3 \mu_{B}^{2}}{4 k_{q} r_{q}^{2} e^{2}}$, $\beta=v / c$ (if $r \approx 10^{-11} \mathrm{~cm}$ then $3 \mu_{B}^{2} /\left(4 k_{q} r_{q}^{2} e^{2}\right) \approx 1$ ).

Thus if to take into account the electric dipole moment of virtual particles pair created by quantum entity in the physical vacuum, this makes it possible to offer a physical explanation to the following experimentally observed phenomenon: the change in the size of a system of electric charges being in equilibrium only under the action of electrostatic forces between the charges when the system is set in motion.
Conclusion. The quantum entity wavelength is determined by the size of electric dipole formed by a pair of electrically unlike virtual particles created by the quantum entity in the physical vacuum. It is noteworthy that in the planetary model of atom the radius of stationary orbits of electrons in an atom, which is equal to $n \lambda$ ( $n$ is the principal quantum number), is a multiple of the size of electric dipole created by electron in the physical vacuum.

\section{The Phase of Wave Function}

It was shown in Section 2 that the wave function frequency of a quantum entity is the spin precession frequency of the pair of virtual particles created by the quantum entity in the physical vacuum. With precession frequency $\omega_{v}$ the precession angle (phase) $\alpha$ is associated, which varies with time as

$$
\alpha=\omega_{v} t .
$$

The distance $\lambda_{v}$ traveled by the quantum entity over the period of precession, $T=2 \pi / \omega_{v}$, is determined as $\lambda_{v}=2 \pi v / \omega_{v}$. Here the quantum entity speed $v$ is the phase speed of propagation of the precession angle (phase) in the space. For the freely moving (in the absence of external forces) quantum entity, taking into account Eqs. (4), (6) and (19), $\lambda_{v}=2 \lambda$. A wave vector $\mathbf{k}_{v}$ associated with the propagation of the precession angle (phase) may be introduced:

$$
\mathbf{k}_{v}=\frac{2 \pi \mathbf{p}}{\lambda_{v} p} .
$$

Using Eqs. (42) and (43), the precession angle can be expressed as a function of positional coordinate $\mathbf{x}$ and time $t, \alpha(\mathbf{x}, t) ; \alpha(\mathbf{x}, t)$ takes values in the range $0 \div 2 \pi$ :

$$
\alpha(\mathbf{x}, t)=\omega_{v} t-\left[\mathbf{x} \frac{\mathbf{k}_{v}}{2 \pi}\right] 2 \pi .
$$

If $\mathbf{x k}_{v} /(2 \pi)$ in Eq. (44) is equal to an integer, Eq. (44) will be expressed as $\alpha(\mathbf{x}, t)=\omega_{v} t-\mathbf{x k}_{v}$. Taking into account Eq. (19), the latter equation for the precession angle (phase) $\alpha(\mathbf{x}, t)$ is similar to the equation for the phase of Schrödinger wave function, $\boldsymbol{\psi}_{S}(\mathbf{x}, t)$, see Eq. (8).

The wave function phase determines interference of quantum entities. The experiments show [13] that the interference pattern derived as a result of passing quantum entities through a crystal grating does not depend on whether all quantum entities arrive at the grating simultaneously or singly, that is, only one quantum entity is present within the crystal grating at a time. In quantum theory this is interpreted as the interference of the quantum entity with itself. In classical physics this phenomenon is seen as a proof of existence of a force between the nucleus of scattering substance and the quantum entity scattered. The force depends on the phase of wave function. It will be shown in this section that the creation of pairs of virtual particles by quantum entities in the physical vacuum results in the emergence of such a force. 
Section 3 discusses the QEDM formed by a pair of virtual particles created by a quantum entity in the physical vacuum. Since the QEDM of virtual particle pair is aligned with the spin of the pair [6], the precession of spin of virtual particles (Section 2) means the precession of the QEDM as well. Accounting for this precession results in that the interaction of QEDM of quantum entity with nuclei of the scattering substance appears dependent on the angles of precession and nutation. Figure 3 is a diagram that illustrates this interaction using as an example a negatively charged quantum entity moving at velocity $\boldsymbol{v}$ in the electric field of nucleus. The QEDM $\mathbf{d}_{v}$ of the pair of virtual particles created by the quantum entity precesses with frequency $\boldsymbol{\omega}_{v}$, which, according to Eq. (19), equals the frequency of the Schrödinger wave function of the quantum entity, $\omega_{S h}$. The precession results in a nonzero projection $\mathbf{d}_{v}$ onto the straight line $\mathbf{r}$ between the quantum entity and nucleus.

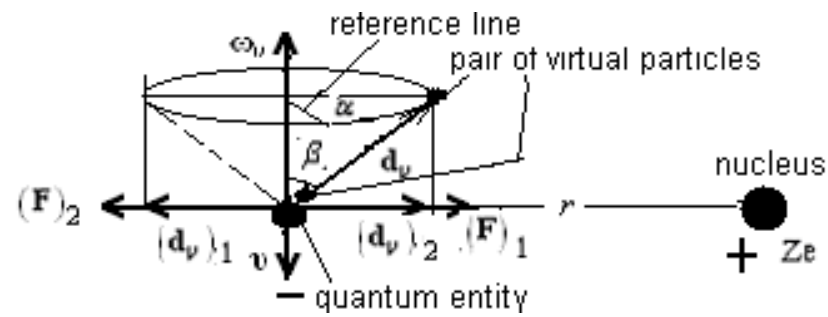

Figure 3. The forces between a nucleus of scattering substance and quantum entity: $(\mathbf{F})_{1}$ and $(\mathbf{F})_{2} ; r$ is the distance between them; $\left(\mathbf{d}_{v}\right)_{1}$ and $\left(\mathbf{d}_{v}\right)_{2}$ are projections of QEDM, $\mathbf{d}_{v}$, of the quantum entity onto $\mathbf{r}$; $\alpha$ is the precession angle (phase) counted off from the reference line normal to the Figure plane; $\beta$ is the nutation angle; $\boldsymbol{\omega}_{v}$ the precession frequency; Ze the nucleus charge; $\boldsymbol{v}$ the velocity of quantum entity

The projections $\left(\mathbf{d}_{v}\right)_{1}$ and $\left(\mathbf{d}_{v}\right)_{2}$ which are greatest in value and directed oppositely are shown on Figure 3. There is a relationship between the quantity $\left(\mathbf{d}_{v}\right)_{1}$ and force $(\mathbf{F})_{1}$, and also between $\left(\mathbf{d}_{v}\right)_{2}$ and force $(\mathbf{F})_{2}$; $(\mathbf{F})_{1}$ and $(\mathbf{F})_{2}$ (in generalized form $\mathbf{F}$ ) are the forces of interaction between the quantum entity and nucleus. Assuming that $\boldsymbol{\omega}_{v} \perp \mathbf{r}$ we obtain the following [9]:

$$
\begin{aligned}
& (F)_{1}=\left(d_{v}\right)_{1} Z e\left(1 /(r-\lambda \sin \beta)^{2}-1 / r^{2}\right), \\
& (F)_{2}=\left(d_{v}\right)_{2} Z e\left(1 / r^{2}-1 /(r+\lambda \sin \beta)^{2}\right),
\end{aligned}
$$

where $\mathrm{Ze}$ is the nucleus charge, $\beta$ is the nutation angle. Assuming that the precession angle (phase) $\alpha$ is counted off from the reference line normal to the Figure plane, we have:

$$
F=\left\{\begin{array}{l}
d_{v} \sin \beta \sin \alpha \cdot Z e\left(-1 / r^{2}+1 /(r-\lambda \sin \alpha \sin \beta)^{2}\right) \\
\text { if } 0 \leq \theta<\pi, \\
d_{v} \sin \beta \sin \alpha \cdot Z e\left(+1 / r^{2}-1 /(r+\lambda \sin \alpha \sin \beta)^{2}\right) \\
\text { if } \pi \leq \theta<2 \pi .
\end{array}\right.
$$

Due to the force between the nucleus and quantum entity, which depends on the precession and nutation angles, an interference pattern may be observed in passing the quantum entity through a crystal grating. Using the expression (44) the condition for producing an interference pattern can be written as follows:

$$
b=n 2 \lambda,
$$

where $b$ is the distance traveled by the quantum entity between the atoms of crystal grating. While the quantum entity is traveling over the distance $b$, the precession angle $\alpha$ changes by $2 \pi n$, where $n$ is an integer (angle $\beta$ is assumed to be constant).

If $(\mathbf{F})_{1} \approx(\mathbf{F})_{2}$ (note that, according to (45) and (46), $(\mathbf{F})_{1}=(\mathbf{F})_{2}$ under $\left.\lambda \sin \alpha \sin \beta=r \sqrt{3}\right)$, the interference pattern may be observed not only under condition (47), but also under condition

$$
b=n \lambda \text {. }
$$

Thus the spin precession angle (phase) of pair of virtual particles created by the quantum entity in the physical vacuum determined by Eq. (45) is a periodically varied quantity, and the spin precession angle (phase) can be considered as a "pilot wave" that accompanies the motion of the quantum entity. In this connection the matter wave of a quantum entity can be termed as the "phase wave". The "phase wave" frequency equals the Schrödinger wave function frequency of the quantum entity, the "phase wave" wavelength equals the twofold wave function wavelength of the entity.

\section{Quantum Correlations}

The phenomenon of quantum correlations is associated with the matter waves: it is correlation of properties of spatially separated quantum entities described by the same wave function. Quantum correlations take place for quantum entities having either zero rest mass (e.g. photon) or nonzero rest mass. It is experimentally established [15] that quantum correlations of two quantum entities take place not only in the process of simultaneous detection of both quantum entities, but also in the case of different times of traveling of the quantum entities from their sources to the detectors. This indicates that quantum correlations are performed in the physical vacuum.

In quantum mechanics, the only explanation to quantum correlations while conserving the cause and effect relationships and while taking into account the role of the physical vacuum is the introduction of unknown "superluminal" forces. It is shown in [6,7] that quantum correlations between quantum entities are effected through spin correlations between pairs of virtual particles created by the quantum entities in the physical vacuum. It is shown as well that the spin correlations may be effected by spin supercurrents whose properties are similar to those of spin supercurrents between spin structures in superfluid ${ }^{3} \mathrm{He}-\mathrm{B}$. The question arises whether the speed of spin supercurrent can exceed the speed of light in vacuum, $c$, which is a speed limit not only for light but for any moving quantum entity with nonzero rest mass?

To answer the question let us discuss the phenomenon which is characteristic of both light and moving particles: 
the increase in mass. According to (20) and (23), the motion of any quantum entity is accompanied by creation of mass and, consequently, by a process in the gravitational field. Therefore, there exists a characteristic speed of quantum entity motion, at least relative to the gravitational field, that is, the speed of light in a vacuum. If the speed of motion of a quantum entity with nonzero rest mass is becoming equal to $c$, then the speed of the pair of virtual particles created by the quantum entity in the physical vacuum becomes equal to the speed of the pair of virtual particles created by the photon in the physical vacuum. Consequently, the motion of this quantum entity will be accompanied by electromagnetic radiation. This phenomenon is observed in the experiments with electrons moving at a superluminal speed and is referred to as Cherenkov's effect [16].

The physical process underlying quantum correlations is the spin supercurrent, which differs fundamentally from the process occuring in the physical vacuum at the motion of quantum entity. A spin supercurrent arises between virtual particles, but it does not produce the latter. Therefore, the spin supercurrent is not accompanied by the birth of a mass and is not a process in the gravitational field. So its speed may be greater than the speed of light in a vacuum.

\section{Conclusion}

The comparison of the characteristics of the wave function of quantum entities and those of the virtual particles pairs created by the quantum entities in the physical vacuum allows one to suggest the following:

- The wave properties of quantum entities are due to creation of pairs of virtual particles by the quantum entities in the physical vacuum.

- The frequency of Schrödinger's wave function is the frequency of precession of spin of the pair of virtual particles created by the quantum entity.

- The wave function wavelength is equal to $2 \pi$-fold size of the electric dipole produced by a pair of virtual particles created by the quantum entity.

- The phase of wave function is associated with the precession angle of spin of the pair of virtual particles created by the quantum entity in the physical vacuum. Quantum correlations of quantum entities are due to spin correlations between pairs of virtual particles created by the quantum entities.

\section{Statement of Competing Interests}

The author has no competing interests.

\section{List of Abbreviations}

QEDM - Quantum Electric Dipole Moment.

\section{References}

[1] De Broglie, L.V, “A Tentative Theory of Light Quanta," Philosophical Magazine, 47. 446, 1924.

[2] Wichmann, E.H, Quantum Physics. Berkeley physics course, v. IV, McGraw-Hill Book company, 1971.

[3] Sinha K.P., Sudarshan E.C.G. and Vigier J.P, "Superfluid Vacuum Carrying Real Einstein - De Broglie Waves", Phys. Lett. 114A, No. 6, 298-300, 1986.

[4] Mandl F. and Shaw G, Quantum Field Theory, John Wiley \& Sons, Chichester UK, revised edition, 56, 176, 1984/2002.

[5] Sedov L.I, A Course in Continuum Mechanics, v. 1-4, WoltersNoordhoff, 1971-1972.

[6] Boldyreva L.B, What does this give to physics: attributing the properties of superfluid ${ }^{3} \mathrm{He}-\mathrm{B}$ to physical vacuum? Moscow, KRASAND, 2012.

[7] Boldyreva L.B, "Quantum correlations - Spin supercurrents", International Journal of Quantum Information, 12, No. 1, 1450007 (13 pp.), 2014.

[8] Born M, Einstein's Theory of Relativity, Dover Publications, New York, 1962.

[9] Purcell E.M, Electricity and Magnetism. Berkeley physics course, v. 2, McGraw-Hill Book company, 1965.

[10] Thomas L.T, "The Kinematics of an Electron with an Axis," Philosophical Magazine 3 (1), 1-22, 1927.

[11] Kroemer H. and Kittel C, Thermal Physics (2nd ed.), W. H. Freeman company, 1980.

[12] Lamoreaux, S.K, "Demonstration of the Casimir Force in the 0.6 to 6um Range," Phys. Rev. Lett. 78, 5-8, 1997.

[13] Capasso F, Munday J.N. and Parsegian V.A, "Measured longrange repulsive Casimir-Lifshitz forces," Nature, 457, 170-173, 2009.

[14] Biberman L., Sushkin N. and Fabrikant V, "Diffraction of successively travelling electrons," Reports of the Academy of Sciences of USSR, LXVI, No.2, 185-186, 1949.

[15] Belinskii A.V, "Quantum nonlocality and the absence of a priori values for measurable quantities in experiments with photons," Physics Uspekhi, 46, 877-881, 2003.

[16] Čerenkov P.A, "Visible radiation produced by electrons moving in a medium with velocities exceeding that of light," Phys. Rev. 52, 378, 1937. 